\title{
Nuclear Fuel Cycle Reasoner: PNNL FY12 Report
}

\author{
R Hohimer Z G Gastelum \\ K Pomiak J Strasburg \\ P Neorr
}

May 2013

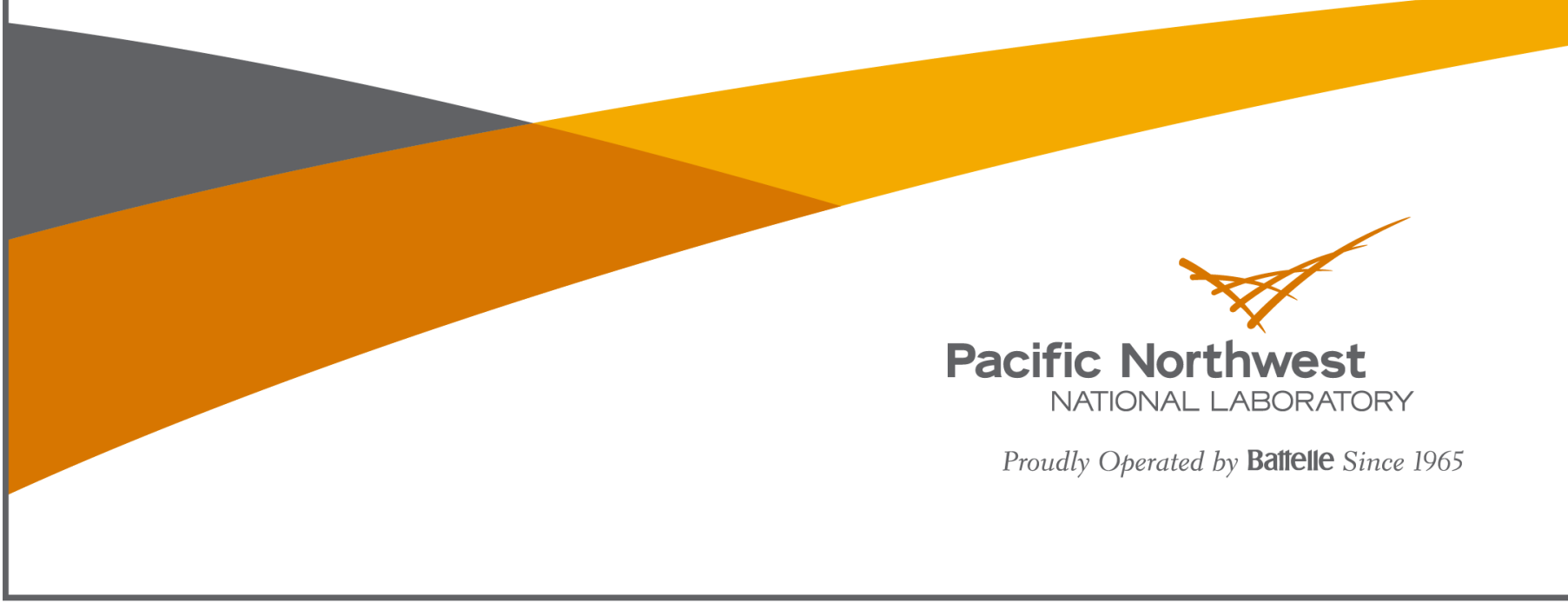




\title{
DISCLAIMER
}

This report was prepared as an account of work sponsored by an agency of the United States Government. Neither the United States Government nor any agency thereof, nor Battelle Memorial Institute, nor any of their employees, makes any warranty, express or implied, or assumes any legal liability or responsibility for the accuracy, completeness, or usefulness of any information, apparatus, product, or process disclosed, or represents that its use would not infringe privately owned rights. Reference herein to any specific commercial product, process, or service by trade name, trademark, manufacturer, or otherwise does not necessarily constitute or imply its endorsement, recommendation, or favoring by the United States Government or any agency thereof, or Battelle Memorial Institute. The views and opinions of authors expressed herein do not necessarily state or reflect those of the United States Government or any agency thereof.

\author{
PACIFIC NORTHWEST NATIONAL LABORATORY \\ operated by \\ BATTELLE \\ for the \\ UNITED STATES DEPARTMENT OF ENERGY \\ under Contract DE-AC05-76RL01830 \\ Printed in the United States of America \\ Available to DOE and DOE contractors from the \\ Office of Scientific and Technical Information, \\ P.O. Box 62, Oak Ridge, TN 37831-0062; \\ ph: (865) 576-8401 \\ fax: $(865) 576-5728$ \\ email: reports $a$ adonis.osti.gov \\ Available to the public from the National Technical Information Service \\ 5301 Shawnee Rd., Alexandria, VA 22312 \\ ph: (800) 553-NTIS (6847) \\ email: $\underline{\text { orders } a n t i s . g o v}<$ http://www.ntis.gov/about/form.aspx > \\ Online ordering: http://www.ntis.gov
}

से This document was printed on recycled paper.

(8/2010) 


\title{
Nuclear Fuel Cycle Reasoner: PNNL FY12 Report
}

\author{
R Hohimer Z Gastelum \\ K Pomiak J Strasburg \\ P Neorr
}

May 2013

Prepared for

the U.S. Department of Energy

under Contract DE-AC05-76RL01830

Pacific Northwest National Laboratory

Richland, Washington 99352 



\section{Summary}

Building on previous internal investments and leveraging ongoing advancements in semantic technologies, PNNL implemented a formal reasoning framework and applied it to a specific challenge in nuclear nonproliferation. The Semantic Nonproliferation Analysis Platform (SNAP) was developed as a preliminary graphical user interface to demonstrate the potential power of the underlying semantic technologies to analyze and explore facts and relationships relating to the nuclear fuel cycle (NFC). In developing this proof of concept prototype, the utility and relevance of semantic technologies to the Office of Defense Nuclear Nonproliferation Research and Development (DNN R\&D) has been better understood.

The Nuclear Fuel Cycle Reasoner project developed a knowledge-driven reasoning framework that incorporates subject matter expertise to enable computer-aided data triage and analysis. Subject matter expertise about the NFC is formally represented in a set of domain-specific conceptual models. Low level information is incorporated as facts into a semantic graph (referred to as "working memory"). A hierarchy of reasoning agents, each programmed with their own unique set of rules, has a "publish and subscribe" relationship with working memory. An individual agent subscribes to and processes a specific subset of facts according to its internally defined rules. Based on the results of this processing, the agents can publish new facts into working memory which can be subscribed to by different agents in the hierarchy. This creates a belief propagation network based on the logic of the subject matter expertise encapsulated in the agents; hence the agents computationally interpret the data as the subject matter experts would. The concepts and facts within working memory could come from multiple data types or intelligence sources (INTs) and the agents can be programmed to reason over multiple domains.

To define and scope the development effort a use case was constructed that embodied the requirements for a reasoning framework. The use case focused on a safeguards analyst who is charged with assessing the status of nuclear activities in foreign countries. In addition to helping a safeguards analyst reason about a nation state's relationship to the NFC, the framework also aids the analyst in understanding the NFC by exploring concepts and the relationships between those concepts. 



\section{Contents}

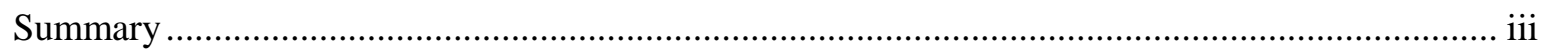

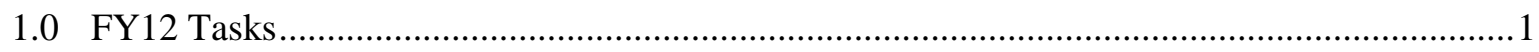

1.1 Use Case

1.2 SNAP - Semantic Nonproliferation Analytic Platform ................................................

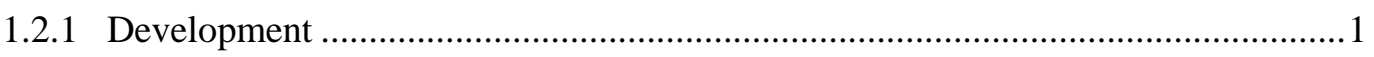

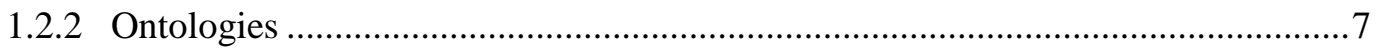

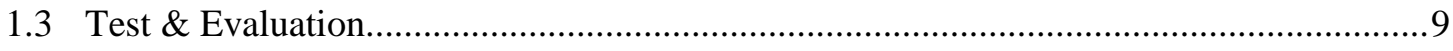

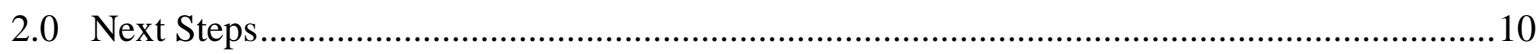

Appendix A Czech Republic Use Case Demonstration ............................................................. A.1

Appendix B STL \& PNNL Early Stage Evaluation Plan for Utility of SNAP ............................ B.1 


\section{Figures}

Figure 1.Reasoning Agents have Access to the Ontologies, the Contextual Knowledgebases, and the Working Memory Semantic Graph.

Figure 2. 1) Domain Ontology, 2) Contextual Knowledgebases, 3) Reasoning Agent Knowledgebases,

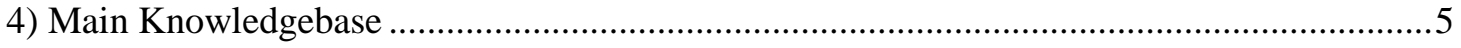

Figure 3. Reasoning Agent have a Publish \& Subscribe relationship with the Working Memory....6

Figure 4. After reasoning two reactors are represented in the semantic graph ...............................7

Figure 5. The Ontologies Encapsulate Knowledge about the Nuclear Fuel Cycle Domain ..............8 


\subsection{FY12 Tasks}

\subsection{Use Case}

A specific use case was identified to focus development of the reasoning framework. Based on discussions with Defense Nuclear Nonproliferation Research \& Development (DNN R\&D) program managers, a use case was developed from the perspective of an IAEA safeguards analyst. The analyst interacts with an application aimed at assisting in the assessment of a nation state's involvement within the Nuclear Fuel Cycle (NFC). The safeguards-related analysis translates specifics of a particular inspection target (in this case, the Czech Republic) to points of relevance in the NFC. The team used the use-case to define the requirements for the development and the scope of the research. A previous report on the Use Case selection was provided in early 2012. (Strasburg JD, RE Hohimer, ZN Gastelum, and JM Kornell. 2012) Appendix A has a transcript of the Use Case scenario that was developed. This script was used to illustrate a new safeguards analyst beginning a new analysis project.

\subsection{SNAP - Semantic Nonproliferation Analysis Platform}

\subsubsection{Development}

An Agile approach was used to develop the SNAP application. The intent was to get a product implemented as quickly as possible and then improve upon it iteratively.

\subsubsection{User Experience}

Early in the development of SNAP the decision was made to implement the necessary functionality (i.e., utility) prior to developing a more sophisticated graphical user interface (i.e., usability). The development of intuitive user interfaces requires a close collaboration with end-users and is beyond the scope of this prototype demonstration. As a result the current SNAP user interface is a development environment suitable for a knowledge engineer but not designed for a safeguards analyst.

The prototype demonstration is designed to show the potential utility of semantic technologies. Should the underlying capability be of interest to a specific end-user, a targeted graphical user interface can be developed to enhance the usability.

\subsubsection{Framework}

In FY10-FY11, PNNL invested internal funds in the creation of a reasoning framework known as Columnar Hierarchical Auto-associative Memory Processing In Ontological Networks (CHAMPION). This framework was conceived in FY10 and an early prototype implemented in FY11.

This hierarchical design and approach to reasoning about facts in a semantic graph was seen as wellsuited for supporting the NFC Reasoning project. (Hohimer et al. 2011)

The choice to use the CHAMPION framework was made based on some specific capabilities required by design. 


\section{Logical Consistency Management}

When reasoning over a semantic graph, a reasoner requires that the semantic graph is logically consistent. If there are logical errors in the graph, the reasoner throws an error and the reasoning process stops. The larger and more diverse the semantic graph becomes, the harder it is to maintain its logical consistency.

The CHAMPION framework does not place a single reasoner over the entire semantic graph. Instead, the framework constructs a hierarchy of reasoners that only reason over well-defined sub-graphs of the larger global semantic graph. Each reasoner in the hierarchy is scoped to reason about a single class of objects defined in the ontologies of the NFC domain.

\section{Composite and Aggregation Recognition}

Typical reasoning frameworks are able to manage subsumptive reasoning relatively well. This is because ontology languages have explicit predicates to specify subsumptive relationships. This research used Ontology Web Language (OWL). OWL has the following specific predicates to reason about subsumption:

- Class

- rdfs:subClassOf

- equivalentClass

- oneOf

- disjointWith

- unionOf

- complementOf

- intersectionOf

With these predicates available to the reasoner, inferences about subsumption are relatively straightforward.

However, the OWL language does not currently include such expressive predicates to entail composition. Due to the lack of these predicates, a reasoner cannot recognize when the necessary and sufficient parts to a whole are present. The primary reason for this is that subsumptive properties are not domain specific, whereas composition properties are very domain specific. Subsumption is about set theory, composition is about domain components.

Since the OWL language does not support composition as well as desired, we developed the support for composition recognition in the framework. The axioms and rules defined in the ontologies allow the reasoners within the framework to recognize when the necessary parts are present to entail the whole.

This kind of composition recognition was considered necessary to reason about the NFC domain. 


\section{Existential Reasoning}

Existential reasoning is closely related to the previous topic of composition. In composition reasoning, when all the necessary and sufficient parts to a whole exist the reasoner in the framework can instantiate a new individual of the appropriate class (e.g., when the parts to a car are recognized, a car can be instantiated).

There are some things that infer that other things exist. Consider a traffic ticket. A traffic ticket is not a car. It is not a part to a car. However, it may be enough evidence, according to the axioms in the ontologies, to infer that a car exists.

\subsubsection{Application}

\section{Components of the Application}

During the development of SNAP the memory management of the knowledge resources evolved. The platform is made up of several semantic graphs (including the ontologies).

- Ontologies

- NFC domain ontology

- Imported ontologies

- Classification rules ontologies

- Knowledgebases

- Main semantic graph (working memory)

- Contextual knowledge graphs (reference facts)

- Local reasoning agent graphs (each agent has a local semantic graph) 


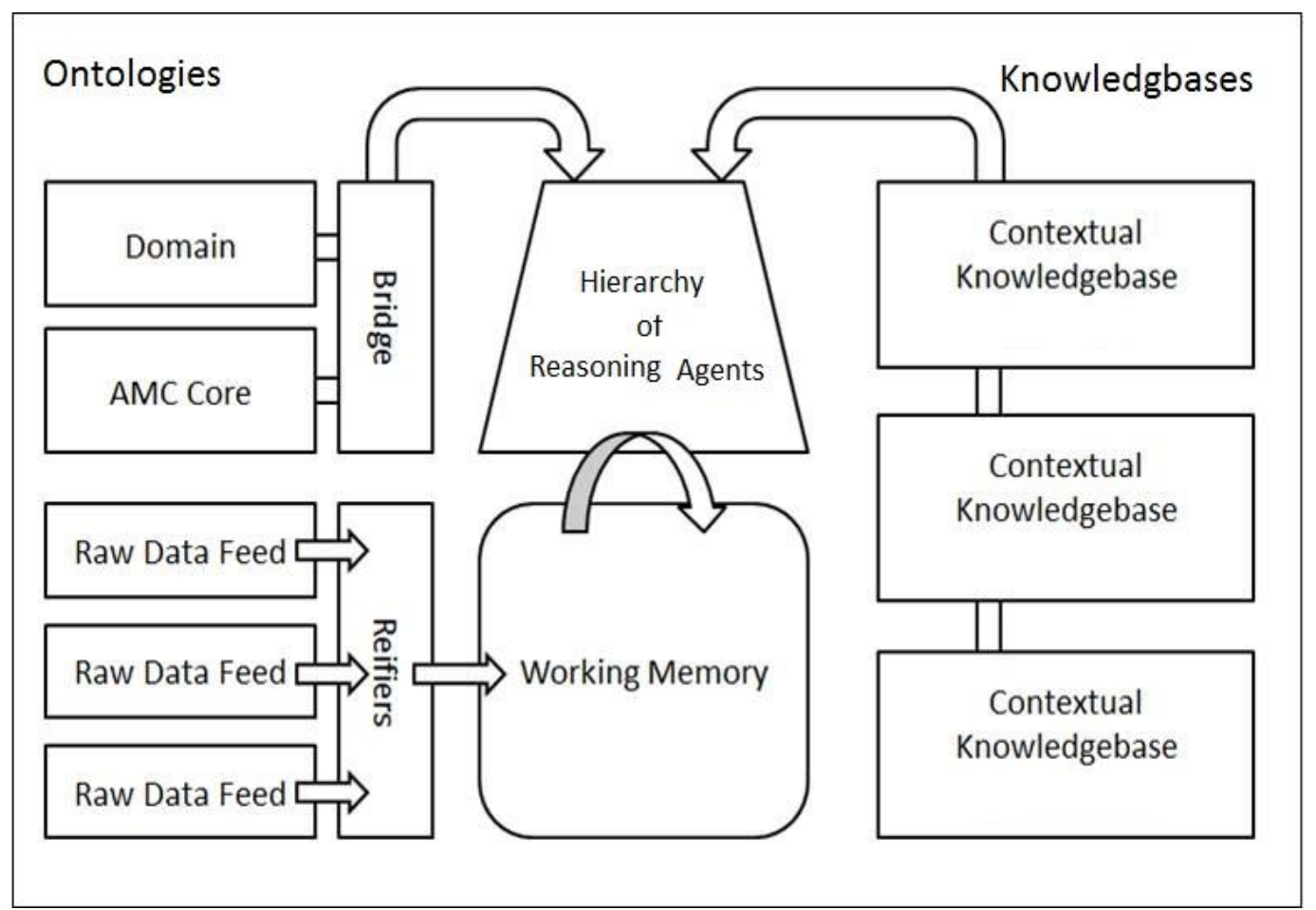

Figure 1. Reasoning Agents have Access to the Ontologies, the Contextual Knowledgebases, and the Working Memory Semantic Graph

The most critical graphs are the local graphs inside each of the reasoning agents. The agents need to be able to access the contents of all the other semantic graphs within the system as shown in Figure 1. 


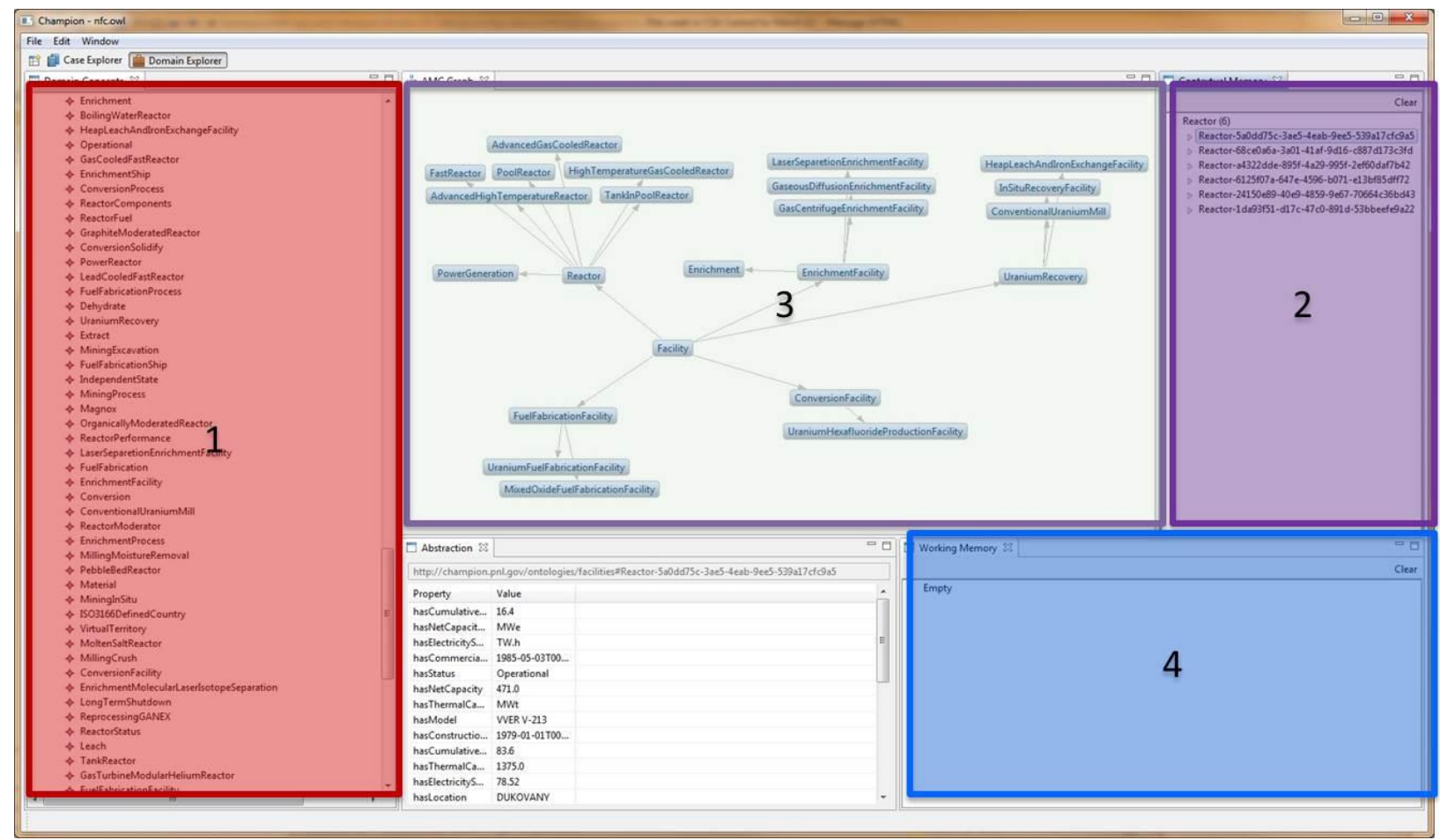

Figure 2. 1) Domain Ontology, 2) Contextual Knowledgebases, 3) Reasoning Agent Knowledgebases, 4) Main Knowledgebase

\section{SNAP Functionality - What the Application Actually Does}

The SNAP application maintains a semantic graph. When new facts are added to this semantic graph a hierarchy of reasoning agents examine the semantic graph and determine if the current state of the graph entails new facts that can be added.

There is a publish and subscribe relationship between the reasoning agents and the working memory semantic graph. When facts are added to the working memory semantic graph any subscribing reasoning agent receives a copy of the facts into their local memory semantic graph. The local semantic graph inside the reasoning agent has the necessary ontologies and knowledgebases loaded so that it can interpret the new facts in the appropriate context. If any new facts are entailed these new facts are added to the main working memory. The figure below shows how an AMC (Power Reactor Reasoning Component) can evaluate an individual (Reactor) and add a new individual (Power Reactor) into the working memory. 


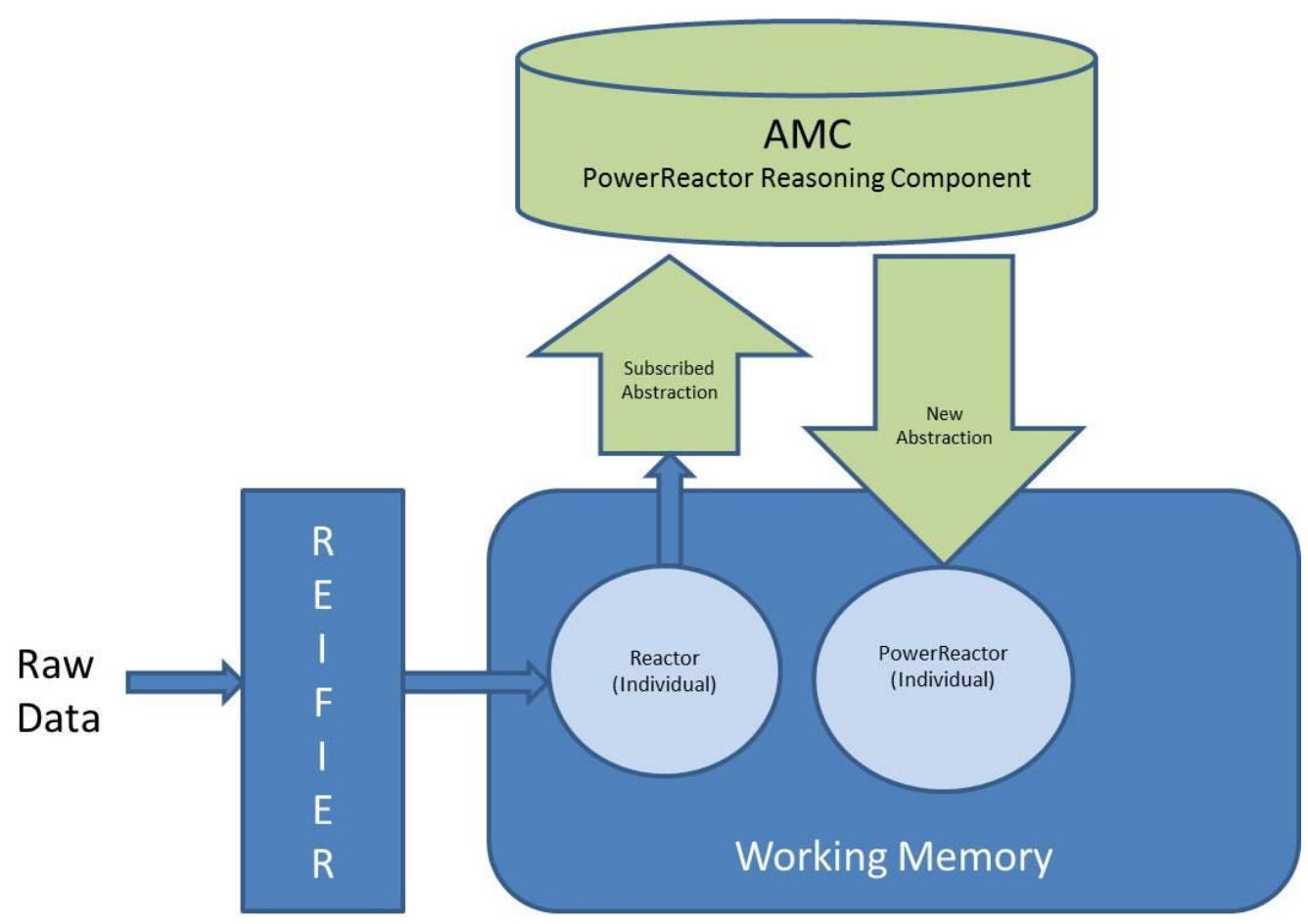

Figure 3. Reasoning Agent have a Publish \& Subscribe relationship with the Working Memory

\section{Queries into Working Memory Semantic Graph}

In the use case, a safeguards analyst is able to add new facts into the working memory. Each time a new fact is added the agents in the reasoning framework examine the state of the working memory and may add additional facts. In order for the user to examine the state of the working memory, the SPARQL language can be used to submit queries. In Figure 4 it is shown that although only a single nuclear reactor was asserted into working memory, after the reasoners have added additional entailments, there are two reactors represented in the working memory (yellow nodes in graph shown). 


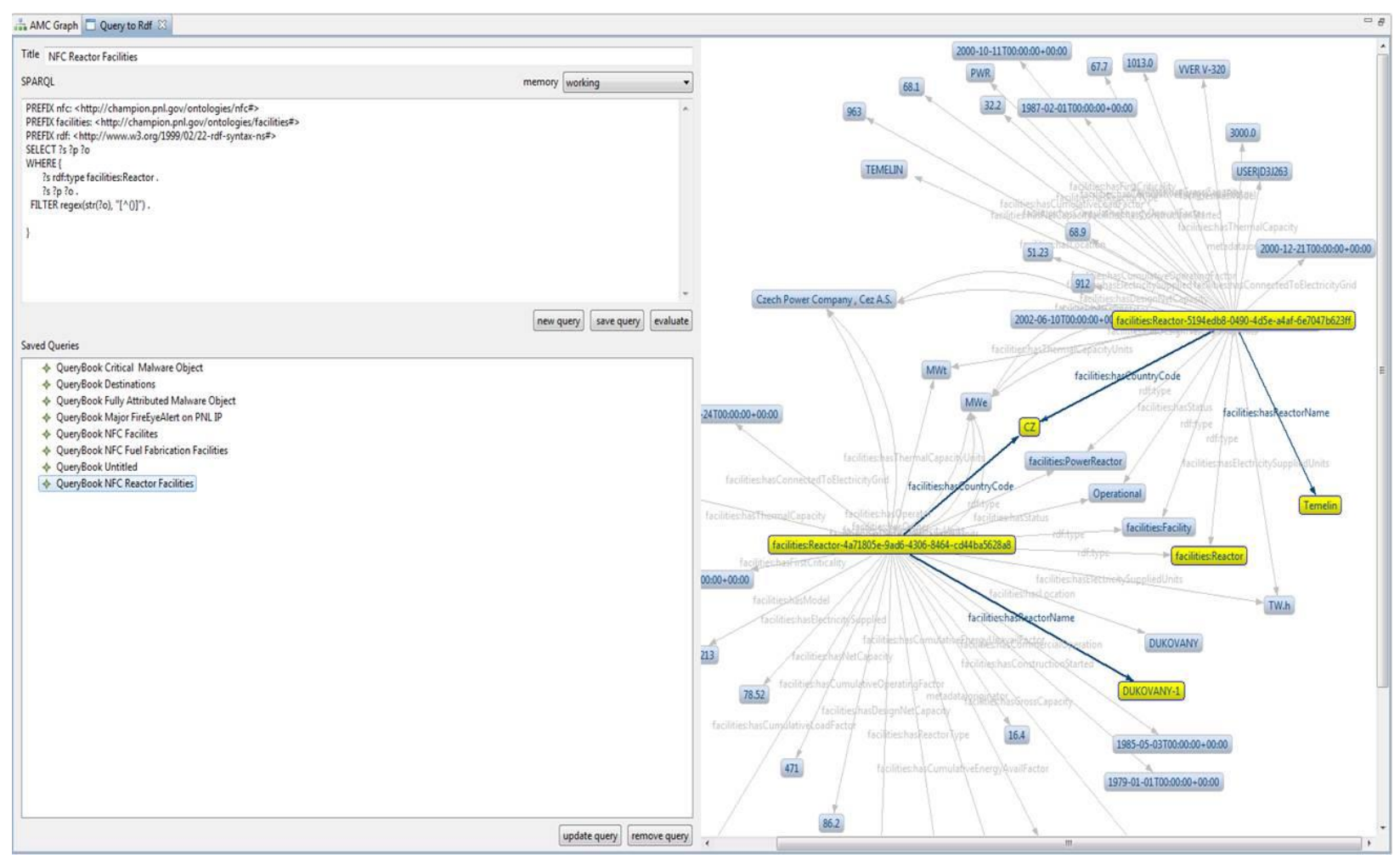

Figure 4. After reasoning two reactors are represented in the semantic graph

\subsubsection{Ontologies}

The hypothesis of the team was that ontologies and reasoning about the NFC could help analysts achieve quicker and more insightful situational awareness about a nation state's involvement with the nuclear fuel cycle processes.

To computationally aid an analyst toward this better understanding a diverse set of ontologies and knowledge-bases were required. Subject Matter Expert (SME) knowledge was needed to understand how to interpret data.

The domain knowledge required to address the safeguards use-case is multi-facetted. The knowledge centric to the NFC was encoded into a file called nfc.owl. This was the file considered the main or controlling ontology.

Other ontologies that contained supporting knowledge were imported into the domain ontology. This import mechanism is more fully described in a following section of this report (see Ontologies for Integrated Modeling). 


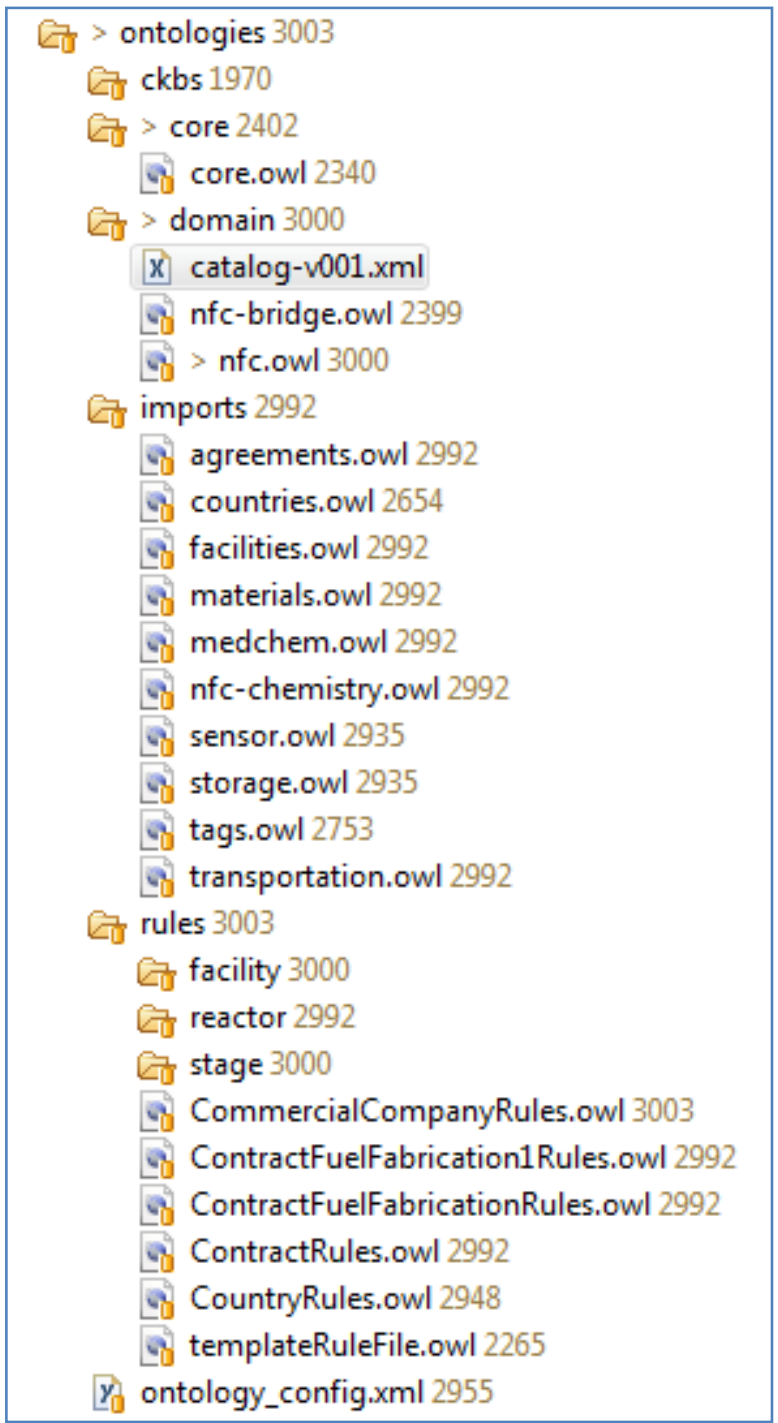

Figure 5. The Ontologies Encapsulate Knowledge about the Nuclear Fuel Cycle Domain

\subsubsection{Strategy for Ontology Development}

An approach adopted for ontology development was to divide the domain space into several parts. The first part was the "broad and shallow" conceptual space of the Freeze Frame graphic. This ontology would contain the concepts of the major steps in the NFC. The second part was an exemplary "deep and narrow" conceptual space. The scope of this ontology would be the supporting information beneath one of the major steps in the broad and shallow ontology.

To clarify with example, the broad and shallow ontology would define the major step of enrichment, and the Deep and Narrow ontology would define the sub-processes required to enrich uranium. 


\subsection{Test \& Evaluation}

At the end of FY12, we considered approaches for evaluating the utility of SNAP as opposed to its usability. Usability is an inherent "user experience" issue and beyond the scope of this proof-of-concept demonstration. Instead resources were spent to develop the utility of a semantically enabled cognitive assistance platform.

The proposed evaluation strategy (documented in Appendix B) utilized semi-structured interviews with selected users from potential problem spaces (e.g., safeguards analysts). The interviews would take the form of execution of a problem space task by the interviewee (e.g., identifying all nuclear facilities in a particular country) without the use of SNAP. The interviewer would then guide the user through the same task using SNAP. The interviewer would solicit feedback throughout both iterations, garnering information regarding both specific user needs and SNAP's ability to meet them. 


\subsection{Next Steps}

The project has successfully developed a platform for ingesting a wide variety of data related to the nuclear fuel cycle and reasoning about that data. While this project focused on a safeguards use case, the platform is versatile and could be adapted to address many different potential user communities (e.g., safeguards analysts, intelligence community, program managers). SNAP demonstrates the "art of the possible." The next step requires interacting directly with the end-users to understand the specific data sources, queries, and user interface that are appropriate for their problem. With a working demonstration, the project emphasis will shift from development to outreach to the technical and user community.

The framework and capabilities were discussed in a number of technical interchanges, including Semantic Technology in Intelligence, Defense and Security in November 2011 and SPAWAR Technology Symposium in March 2012. (Hohimer and Strasburg 2012) The underlying CHAMPION framework was also presented as part of Science of Multi-INT Workshop in September 2012, using internal PNNL funding. (Hohimer 2012)

\subsection{References}

Hohimer RE, FL Greitzer, CF Noonan, and JD Strasburg. 2011. "CHAMPION: Intelligent Hierarchical Reasoning Agents for Enhanced Decision Support." In Proceedings of the Sixth International Conference on Semantic Technologies for Intelligence, Defense, and Security, Fairfax, Virginia, vol. 808, ed. PCG Costa and KB Laskey, pp. 36-43. CEUR Workshop Proceedings, Aachen, Germany. PNNL-SA-82834, Pacific Northwest National Laboratory, Richland, Washington.

Strasburg JD, RE Hohimer, ZN Gastelum, and JM Kornell. 2012. Nuclear Fuel Cycle Reasoner: Topic Survey and Use Case. PNNL-21151, Pacific Northwest National Laboratory, Richland, Washington.

Hohimer RE, and JD Strasburg. 2012. "CHAMPION: Semantic Technology Resource for Analysis \& Enhanced Decision Support.” Presented at AFCEA/SPAWAR Semantic Technologies Symposium, North Charleston, South Carolina. PNNL-SA-86463, Pacific Northwest National Laboratory, Richland, Washington.

Hohimer RE. 2012. "CHAMPION: A Semantic Reasoning Engine for Triaging Multi-INT Data." Presented at the Science Of Multi-INT (SOMI) Workshop, Chantilly, Virginia. PNNL-SA-90445, Pacific Northwest National Laboratory. 


\section{Appendix A}

\section{Czech Republic Use Case Demonstration}





\section{Appendix A}

\section{Czech Republic Use Case Demonstration}

Jana: Welcome

Intro to the project and team

Zoe: My background at the IAEA as an open source analyst. What we did, and what our problems were: Most analysts were social scientists. Some of the analysts were less familiar with the NFC than others. Some of the open source analysis tasks were technical in nature. Even for the less technical tasks, collection and analysis of data was done manually, without the use of analytical tools. This is quite difficult to do. This lead to the development of the use case to support open source analysis for safeguards verification.

For our use case, we chose the Czech Republic. CR is an interesting example because it has some nuclear fuel cycle capabilities, such as uranium mining and nuclear power plants, but does not have the full fuel cycle. Let's start by having SNAPPER bring in everything it already knows about the CR. The work that is going on under the hood represents a task that every safeguards analyst would perform when starting work on a new country. That is, finding out everything we already know. In this case, SNAPPER is harvesting information from two open IAEA databases: the Power Reactor Information Service, and the Integrated Nuclear Fuel Cycle Information Service.

Ryan: Bring in everything that it knows about Czech Republic

- Load in the PRIS reactors to Contextual memory

- Load other NFCIS facilities to Contextual memory

Zoe: We've brought everything together and automated relevant data collection and integrated it into one knowledge base. From here we can explore the information in Contextual memory

- Can see we have six reactors

- Reactor type

- Vendor

- Thermal output

Ryan: open up one of the reactors and look at properties

Zoe: $\quad$ But these are just the facts from the IAEA database. An SME would interpret these facts. In order to understand the bigger picture, let's have SNAPPER process this information and make additional inferences. Let's run it on one reactor and see what it knows. Let's take, for example, the Temelin 1 reactor. I am partial to this one, since I visited it last year.

Ryan: Assert Temelin 1 to working memory. 
Zoe: We assert the reactor. SNAPPER reasons it is a power reactor, therefore it generates electricity. Given that, we know it is burning nuclear fuel. If we open up the fuel fabrication tab here, we can see there is no fuel fabrication facility support that CR's fuel consumption. If there was one there, we wouldn't necessarily know that it was supplying domestic fuel, but because there isn't a facility there, we can reasonably say that $\mathrm{CR}$ is getting their commercial nuclear fuel from abroad. But we don't know where the fuel is coming from.

As an SME I know that the Czech Republic and Russia have trade agreements and that Russia may be providing fuel. I don't know about Russia-I want to do a quick fact/sanity check

Ryan: In Contextual memory, Show me the Russian Fuel Fabrication facilities that could have generated that fuel for this reactor.

Zoe: So, I want to bring in information about the Russian NFC. And then sort them to bring out those fuel fab facilities that have the capability to produce fuel needed for CR's plants. Again, this is just information from the IAEA databases.

Seeing that Russia has two facilities that could have reasonably produced this fuel, I am more confident that Temelin's fuel came from Russia. As an IAEA analyst, I would also have information to states' safeguards declarations where I could verify that information as well, potentially identifying which of the three plants fabricated Temelin 1's fuel. For now, let's just go ahead by saying that Russia provides the fuel fabrication services for Temelin 1.

Ryan: Assert that Fuel Fabrication facility as a part of the Czech fuel cycle.

Zoe: NFCR can reason over that new information, that Russia is providing that service, and discover that you still don't have enrichment, but you could continue to follow the chain and so on...This is the analytical process of iteratively filling gaps, and asking new questions.

Once an analyst is finished working with a country or a piece of information, with SNAPPER, we also have the ability to SAVE and Share. This is especially useful to communicate results among team members - this is currently done by sharing documents in a group folder, in which the provenance and the analysis can be lost, or on a SharePoint, the workflows of which are still being established. With the capability to save analysis work done in SNAPPER, there is also a capability to share them with remote users. Given the IAEA's rotation policy of $60 \%$ of professional staff rotating out every seven years (and some even more often than that), this could also serve as a useful knowledge retention tool. It also provides a way to share additional information. For example, within the Russian fuel fabrication facility we have asserted into the SNAPPER system, there is a url provided by the IAEA database, which the user can connect to.

\section{Ryan: Open up the Russian fuel fab website}

Zoe: That is what we call the integrated knowledge base. But we can also show information from Pretege, which, once the system is finalized, will all be under a single user interface. It's the same ontology and knowledge base, but a different way to look at it.

The previous capability has shown how the SNAPPER system can compile and reason over information from IAEA databases. But, a larger part of the safeguards analysts' day, and one that is much 
more technically demanding, involves the review of scientific publications. One of the tasks of a safeguards analysts is to review all scientific publications available to the IAEA that are relevant to the nuclear fuel cycle (this is primarily accomplished through WebofScience, Science Direct, and the International Nuclear Information System (INIS, an IAEA maintained database). Bearing in mind that these are primarily social scientists conducting the research and analysis, one could imagine how it might be difficult when the analysts - especially a new, or less fuel cycle saavy analyst- is faced with a publication on the solvent extraction of americium and uranium from a nitrate solution using synergistic mixtures of nitrogen tridentate heterocyclic extractants and chlorinated cobalt dicarbollide, and asked whether it is relevant to the country's nuclear fuel cycle.

For this reason exactly, SNAPPER has the capability to search and reason over the fuel cycle in a country agnostic way. For example, if a safeguards analyst (based on a predefined search string they are provided by their superiors) is finding repeated references to 30B cylinders, he or she can perform a search in the NFCR knowledge base to see what NFCR knows about that container. For example, let's say I am potentially interested 30B cylinders, and I want to determine if they are relevant to the nuclear fuel

Ryan: Enter search for $30 B$ cylinders in ontograph view.

Zoe: Ah, yes. We can see here that 30B cylinders are used to store UF6. I know, from my own experience, that UF6 is used as the feed product for uranium enrichment. But, let's just take a look to see where else we can find UF6 in the nuclear fuel cycle.

\section{Ryan: Expand UF6 in ontograph}

Zoe: $\quad$ Based on the search results, I can see that SNAPPER knows UF6 is also a key material in wet conversion. If I decided that this was a path I would be interested in pursuing further research on related to CR's fuel cycle, I would want to know some of the other materials that are used in that process, to see what else I should be looking for. So let's ask SNAPPER what other chemicals are used in wet conversion...

Ryan: In query part with all the lists, search "Is used in process wet conversion"

Zoe: Ok, that's too many chemicals. To simplify this, let's only look at those chemicals that are radioactive.

Ryan: Modify search to add "and radioactive chemical"

Zoe: $\quad$ This gives us a more reasonable list of research topics to pursue.

Going back now to UF6, what are some other things that I could be considering when looking for potential ties in with CR's nuclear activities? One thing that I could look for is different detection techniques.

Ryan: do the search for detectors for UF6, or explore the UF6 knowledge 
Zoe: I can see from this that UF6 can be detected by radiation detectors, and fluorine detectors. Just like the other analytical topics we discussed today, the analyst could potentially follow down this path, asking more questions, and finding new relevant information.

At this point, we have seen examples of how SNAPPER can work for two different safeguards analytical scenarios. With that note, I will turn things back over to Jana.

\section{Jana: Conclusion remarks}




\section{Appendix B}

\section{STL \& PNNL Early Stage Evaluation Plan for Utility of SNAP}





\section{Appendix B}

\section{STL \& PNNL Early Stage Evaluation Plan for Utility of SNAP}

\section{B.1 Semi-Structured Interviews for Utility Evaluation}

In this current stage of SNAP development, we desire to perform an evaluation focusing on the utility of SNAP, as opposed to its usability. Usability is an inherent "User Experience" (UX) issue and we have not allocated resources to address the UX issues in this first phase. However, we have spent resources to develop the utility of a semantically enabled cognitive assistance platform.

As such, our evaluative strategy will utilize semi-structured interviews with selected users from potential problem spaces (e.g., safeguards analysts). The interviews will take the form of execution of a problem space task by the interviewee (e.g., identifying all nuclear facilities in a particular country) without the use of SNAP. The interviewer will then guide the user through the same task using SNAP. The interviewer will solicit feedback throughout both iterations, garnering information regarding both specific user needs and SNAP's ability to meet them.

The semi-structured interview format is ideal for this evaluation because it places a greater emphasis on the interviewee's point-of-view. This format is more flexible than a structured interview, allowing the interviewer to ask follow-up questions and to change the order of topics and questions for the interview based upon the respondent's answers. It enables the interviewee to provide detailed answers which may deviate from the set of information the interviewer expected to receive, but which reflect the interviewee's perspective on information of relevance. This is crucial in our evaluation effort because we want to ensure that SNAP is providing potential users with the functionality that they deem important.

We are indeed focusing on a qualitative evaluation rather than quantitative. This correlates with validation testing of our software. Namely, we intend to evaluate whether the software is doing what we want it to (and what the potential user base needs it to). In this phase of evaluation, we do not intend to perform verification testing, i.e., ensuring that the software is correctly doing what we want it to. Our initial evaluation is not intended to quantify the performance of SNAP using standardized metrics. Rather, we seek to elicit qualitative measures of usefulness of the underlying data model through interviews with subject matter experts in relevant user fields.

Once the user interface is further developed in the tool, more comprehensive evaluation strategies can be developed. These future strategies can be designed to benchmark performance with and without SNAP using quantitative metrics.

\section{B.2 Development Roadmap}

The June demonstration will be the departure point for further development of both content and platform. From this point, changes should extend or enhance the user story we have started to tell:* 
A new analyst has been assigned a country and wants to come up to speed on the nuclear status of that country using SNAP. The analyst's initial investigation begins with the following inquiries:

- "What NFC-related facilities exist?"

- "What do we know about those facilities?"

- "What are the implications or inferences that we can make from that?"

- "Where are potential gaps in information?"

- e.g., "Where is the Czech Republic's fuel being enriched?"

- "Where are potential gaps in understanding?"

- e.g., "What are 30B cylinders?," "Where is UF6 used in the NFC?," "What other chemical compounds are associated with this process?"

- How do I learn more?

As content is developed in the following areas, the initial scope of that content should include that which is necessary to extend or enhance this user story.

- nuclear fuel cycle chemistry, with related radiological (medical) chemistry

- thorium fuel cycles

- facilities

- facilities internal dynamics

- materials and processes

- sensing and spectroscopy

- containers

- tags, seals, and tamper-proofing

- transport

- chain of custody

- treaties and agreements.

*The "script" from the June 20 demonstration is included in "Appendix B" of this document for reference.

\section{B.3 Goal}

We want to, as far as appropriate, solicit and utilize early independent evaluation of the utility of SNAP. While substantial evaluation is both premature and too costly for current scope, a set of semistructured interviews with potential SNAP users will provide early indication of strengths, weaknesses, and areas for further development. These semi-structured interviews may eventually contribute to controlled evaluations of SNAP. 


\section{B.4 Protocol}

An interview guide should be developed which outlines the process, coverage areas, and primary questions for the interviews. It should include, and expand upon, the following protocol. Structure should be inherent in the guide so as to ensure each interview covers the full evaluation of SNAP that we desire at this juncture of development, and so that SNAP can be qualitatively assessed by each potential user across the whole narrative that we have developed. This will ensure that we receive information that can be correlated on some level for our analysis of the results of this evaluation.

At the same time, the guide must not be rigid, but rather flexible enough to allow for detailed and varied interviewee feedback. Questions should be concise and pointed, yet open-ended to allow interviewee room for exposition and evaluative comments. The interviewer is encouraged to use probing questions to elicit further explanation and clarification from an interviewee's response.

Working from our current story, the interviewer should guide the interviewee through this narrative twice via a series of questions.

In the first iteration, the emphasis for the interviewer is to gain more understanding of the specific use cases where SNAP could be implemented. We want to fully capture the process a potential user (e.g., safeguards analyst) would go through so that we might evaluate whether there are further areas where SNAP can enhance a user's capabilities.

It would be helpful for the interviewer to lead the interviewee along a narrative that is both abstract and specific. Namely, the interviewer should ask more general questions, such as, "If you were a safeguards analyst who was assigned a new country, what would be you first steps in investigating that country?" Additionally, it may aid the interviewee in providing quality, detailed feedback if she is prompted with specific questions at certain points, such as, "What would be your next step if you discovered 'Country $\mathrm{X}$ ' is consuming fuel but has no fuel fabrication facilities?"

The following are example questions which could be included in this narrative interview.

- "If you were a safeguards analyst assigned a new country, what would be your first steps in investigating that country?"

- "What specific information would you look for regarding that country?"

- "What types of (or specific) sources would you go to for that information?"

- "How would you collect and correlate that information?"

- "What other background information (e.g., fuel cycle knowledge) would be helpful in analyzing this information?"

- "What questions would you ask regarding this country?"

- "Would certain information (or lack of information) give you pause or raise questions for you?"

- "What steps would you take to investigate further?"

- "What would you do if you discovered an entity (e.g., chemical compound) or concept (fuel cycle step) you did not understand?" 
The interviewer should feel free to ask probing questions to clarify the respondent's answers or elicit further information.

Following the first iteration, the interviewer should lead the interviewee through a second iteration of the narrative using the information that was established in the first iteration. The focus of the second iteration is to present the respondent with the capabilities of SNAP and to elicit feedback on its utility in enhancing her ability to complete the steps outlined in the first iteration.

The interviewer should show specific features of SNAP, using the June 20 presentation as a guide, and elicit feedback from the respondent on whether that functionality is helpful or not within her process.

Examples include the following:

- Load all Czech Republic reactors and other fuel cycle facilities into memory

- Ask interviewee if this consolidation of information aids in doing an initial assessment of what is already known about a particular country.

- Assert reactor (e.g., Temelin 1) into working memory-have SNAP reason with this

- Ask interviewee if this provides an added benefit to analyzing and correlating data that she collects.

At each relevant step, the interviewer should model a SNAP functionality that can aid the user in the steps of the established narrative. The interviewer should use open-ended questions after each step to draw detailed evaluative feedback from the respondent. If the interviewee indicates that the SNAP feature would be a benefit, as questions such as "Why is this helpful?" and "How does this enhance your abilities?" and even "How could this be improved?" If the respondent indicates that the modeled feature provides no added benefit for them, pursue similar questioning to garner quality feedback for user utility of SNAP.

FORMAT: The interviewer should complete approximately six interviews with six different subject matter experts in the safeguards analysis domain. Each interview should be conducted via Microsoft Lync so that all audio and screen interactions with SNAP can be recorded for evaluation and analysis.

\section{B.5 Expected Result}

This initial evaluation will allow us to begin collecting user comments regarding

- breadth and depth of coverage

- where SNAP (initially) seems to most, or least, amplify analyst effectiveness.

These semi-structured interviews will help guide the development of more elaborate scenarios where it may be possible to conduct evaluations of SNAP by comparing to a control. 



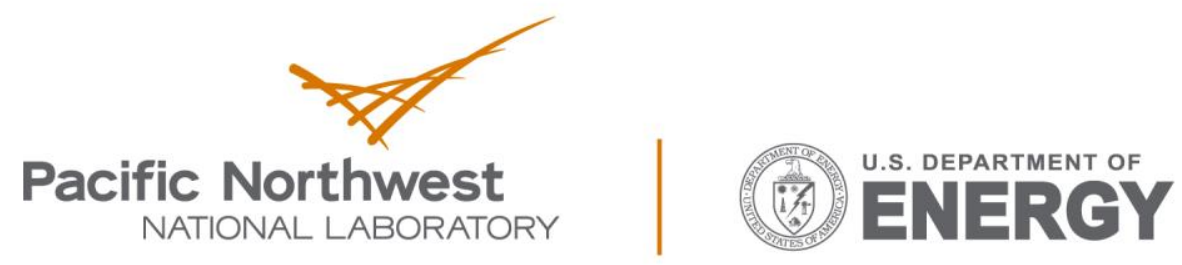

Proudly Operated by Battelle Since 1965

902 Battelle Boulevard

P.O. Box 999

Richland, WA 99352

1-888-375-PNNL (7665)

www.pnnl.gov 